\title{
Impact des Caractéristiques Individuelles et des Caractéristiques de la classe sur le concept de soi des élèves de quatrième année Primaire en République Démocratique du Congo
}

\author{
Gratien Mokonzi Bambanota, PhD \\ Service de Planification et d'Évaluation en Éducation, \\ Université de Kisangani, République Démocratique du Congo \\ Jan Van Damme, PhD \\ Bieke De Fraine, PhD
}

Center for Educational Effectiveness and Evaluation, KU Leuven, Belgique

Doi:10.19044/esj.2019.v15n22p56 URL:http://dx.doi.org/10.19044/esj.2019.v15n22p56

\section{Résumé}

La recherche sur l'efficacité de l'école a souvent été critiquée à cause de sa concentration excessive sur les aspects cognitifs de l'éducation, négligeant ainsi les aspects non cognitifs pourtant pas moins importants. Aussi cette étude analyse-t-elle l'impact des caractéristiques individuelles et des caractéristiques de la classe sur le concept de soi scolaire. Ce dernier a été mesuré suivant deux dimensions : le concept de soi en lecture et le concept de soi en mathématiques. L'étude est basée sur un échantillon de 4787 élèves de quatrième année primaire fréquentant 80 classes et 49 écoles de deux villes de la République Démocratique du Congo. Les données ont été collectées à l'aide $\mathrm{du}$ questionnaire de renseignements sur l'élève, du questionnaire d'autodescription et des tests de lecture et de mathématiques. Elles ont été traitées par l'analyse multiniveau multivariée. Les résultats montrent que le concept de soi mesuré au début de l'année est un bon prédicteur du concept de soi mesuré à la fin de l'année scolaire. Les résultats montrent aussi que le niveau cognitif de l'élève influe positivement sur le concept de soi scolaire. Au niveau de la classe, seuls l'expérience professionnelle de l'enseignant et le score moyen obtenu par les élèves à l'échelle du rapport à l'école affectent significativement le concept de soi en lecture. Les limites et les perspectives de l'étude sont également discutées dans cet article.

Mots-clés: Concept de soi, Concept de soi scolaire, Concept de soi en lecture, Concept de soi en mathématiques, Multiniveau, République Démocratique du Congo 


\title{
The Impact of Individual and Class Characteristics on the Self-concept of the Fourth Grade Students of Elementary School in the Democratic Republic of the Congo
}

\author{
Gratien Mokonzi Bambanota, PhD \\ Service de Planification et d'Évaluation en Éducation, \\ Université de Kisangani, République Démocratique du Congo \\ Jan Van Damme, PhD \\ Bieke De Fraine, PhD
}

Center for Educational Effectiveness and Evaluation, KU Leuven, Belgique

\begin{abstract}
Research on school effectiveness has often been criticized for its excessive focus on the cognitive aspects of education, thus neglecting noncognitive aspects that are not less important. This study therefore analyzes the impact of individual and class characteristics on academic self-concept. The latter was measured in two dimensions: self-concept in reading and selfconcept in mathematics. The study is based on a sample of 4787 fourth form pupils of elementary school attending 80 classes and 49 schools of two towns in the Democratic Republic of the Congo. The data have been collected by means of a background questionnaire on the pupil, a self-description questionnaire, reading and mathematics tests. They have been analyzed through multilevel multivariate analysis. The results show that the selfconcept measured at the beginning of the year is a good predictor of the selfconcept measured at the end of the school year. The results show also that the cognitive level of the pupil has a positive impact on academic self-concept. At the class level, only the teacher's professional experience and the average grade obtained at school scale significantly affect self-concept in reading. The limits and prospects of the study are also discussed in this article.
\end{abstract}

Keywords: Self-concept, Academic self-concept, Self-concept in reading, Self-concept in mathematics, Multilevel, Democratic Republic of the Congo

\section{Introduction}

L'efficacité de l'éducation intéresse, depuis les années 1980, aussi bien les décideurs, les chercheurs, les praticiens de l'éducation que les usagers des 
systèmes éducatifs. En effet, au-delà de la réalisation de l'objectif de la scolarisation universelle, il faut "améliorer sous tous ses aspects la qualité de l'éducation dans un souci d'excellence de façon à obtenir pour tous des résultats d'apprentissage reconnus et quantifiables - notamment en ce qui concerne la lecture, l'écriture et le calcul, et les compétences indispensables dans la vie courante"(UNESCO, 2015, p. 189). Souvent associée à la qualité de l'éducation, l'efficacité est généralement définie comme le degré auquel le niveau désiré des résultats est atteint (Scheerens \& Bosker, 1997) ou encore comme l'adéquation entre les résultats atteints et les objectifs escomptés. Elle dépend de plusieurs facteurs dont l'étude ne peut être correctement menée que si l'on tient compte du caractère hiérarchique des systèmes éducatifs. Dans ces systèmes, les élèves subissent l'influence des classes (ou maîtres) qui sont elles-mêmes sous l'effet des écoles (Creemers \& Kyriakides, 2010 ; Palardy \& Rumberger, 2008 ; Scheerens, 1990).

Les variables de l'élève qui sont associées à l'efficacité de l'éducation relèvent de ses caractéristiques démographiques, familiales, scolaires et affectives. Il est en effet reconnu que les émotions et les perceptions entrent en jeu dans les apprentissages (Pekrun, 1992 ; Pekrun et al., 2002 ; Sénéchal, 1997). Parmi les caractéristiques affectives qui ont fait l'objet d'études, le concept de soi occupe une place de choix (Ghazvini, 2011). Entendu comme les perceptions que l'individu a de ses compétences, de ses valeurs, de ses préférences, de ses buts et de ses aspirations (Raynor \& McFarlin, 1986), le concept de soi est une variable multidimensionnelle qui comporte une structure hiérarchique. Il comprend, en plus d'un construit global, une dimension scolaire et une dimension non scolaire.

En général, le concept de soi influe positivement sur la réussite scolaire (Alsker, 1989 ; Altmann \& Dupont, 1988 ; Chiu \& Klassen, 2010 ; Fin \& Ishak, 2014 ; Keltikangas-Järvinen, 1992 ; Kurtz-Costes, 1994 ; Lyon \& MacDonald, 1990 ; Marsh, 1984 ; Marsh, Hau, \& Kong, 2002 ; Uysal, 2015). Toutefois, le concept de soi n'est pas seulement un facteur, il est aussi une variable dépendante qui subit l'effet des facteurs tels que l'âge, le genre, les expériences scolaires passées et la motivation à participer à l'éducation (Michie, Glachan, \& Bray, 2001).

Dans cette étude, nous analysons, sur la base du modèle multiniveau, les caractéristiques de l'élève et de la classe qui affectent le concept de soi scolaire. Ce dernier renvoie à la manière dont un élève considère ou perçoit son propre rendement ou sa propre compétence.

\section{Revue de la littérature}

Le concept de soi

En dépit du nombre important d'études effectuées, il n'y a pas d'unanimité sur la définition du concept de soi, étant donné les différentes 
perspectives théoriques sur lesquelles s'appuient les auteurs (Ghazvini, 2011). Cependant, au-delà de leurs divergences, les auteurs s'accordent sur la nature multidimensionnelle du concept de soi (Marsh, 1990 ; Rotatori, 1994 ; Yeung, Chui, \& Lau, 1999 ; Yeung, McInerney, \& Russell-Bowie, 2001). Ce dernier comprend une variété de dimensions dont certaines sont liées aux aspects de la personnalité (physique, social, émotionnel) et d'autres à la réussite scolaire. D'après Marsh et Yeung (1997), le concept de soi est une série de perceptions ou de points de référence que les individus ont d'eux-mêmes; une série de caractéristiques, d'attributs, de qualités et de déficiences, de capacités et de limites, de valeurs et de relations descriptifs de leur identité.

Pour Ghazvini (2011, p. 1035) :

le concept de soi est un condensé de sept aspects fondamentaux: il constitue une dimension psychologique; il est multidimensionnel; il a une organisation hiérarchique (un concept de soi général et des concepts de soi spécifiques); il est stable, mais à mesure que l'on descend dans la hiérarchie, le concept de soi devient plus spécifique et plus susceptible de changer; ses différentes facettes deviennent plus différenciées avec l'âge et l'expérience; le concept de soi comprend à la fois une description et des aspects évaluatifs; on peut le différencier des autres concepts auxquels il est lié, tel que la performance.

Shavelson, Hurber et Stanton (1976) sont parmi les premiers auteurs qui ont élaboré un modèle conceptuel du concept de soi. Ce modèle place le concept de soi global au sommet de la représentation hiérarchique. Le concept de soi global est divisé en concept de soi scolaire et en concept de soi non scolaire. Le concept de soi scolaire est, à son tour, fractionné en concepts de soi spécifiques se rapportant aux matières scolaires. De même, le concept de soi non scolaire est subdivisé en concept de soi social, émotionnel et physique, chacun décomposé en concepts de soi spécifiques.

C'est en référence à ce modèle que divers instruments de mesure du concept de soi ont principalement été construits. Tel est le cas du "Self Description Questionnaire I" (Marsh, 1988) exploité dans cette étude.

\section{Les facteurs du concept de soi scolaire}

Même si les recherches ont fondamentalement considéré le concept de soi comme un déterminant de la réussite scolaire (Cokely, 2000 ; Eguavoen \& Eniola, 2016 ; Hanan, Shabana, \& Mona, 2017 ; Jirdehi et al., 2018 ; Spinath et al., 2006) quelques études ont également traité ce concept comme une variable dépendante (Audrit, 2015-2016; Tripathy \& Srivastava, 2012) et d'autres encore ont analysé la relation réciproque entre le concept de soi et la réussite scolaire (Awad, 2007 ; O’Mara \& Marsh, 2006 ; Skaalvik \& Hagtvet, 1990). 
$\mathrm{Au}$ vu des résultats des travaux consacrés à l'étude de la relation entre le concept de soi et la réussite scolaire, Marsh et Yeung (1997) insistent sur le besoin de distinguer quatre patterns possibles :

1) La réussite scolaire détermine le concept de soi. Pour ce modèle, les expériences scolaires de succès ou d'échec affectent de manière significative le concept de soi de l'élève.

2) Les niveaux du concept de soi déterminent le degré de réussite scolaire. Suivant ce modèle le concept de soi impacte les niveaux de réussite scolaire.

3) Le concept de soi et la réussite scolaire s'influencent mutuellement.

4) Il existe des variables additionnelles pouvant être à la fois la cause du concept de soi et de la réussite scolaire, parmi lesquelles se trouvent des variables personnelles et environnementales, scolaires et non scolaires.

Analysant les déterminants du concept de soi, Audrit (2015-2016) a relevé trois types de facteurs : (i) les pratiques éducatives, (ii) le cadre familial et (iii) l'intégration sociale. Fait partie des pratiques éducatives la considération que les enseignants ont de leurs élèves. Grâce aux récits de vie récoltés auprès des adolescents inscrits dans un enseignement spécialisé, Audrit (2015-2016, p. 75) a remarqué que "l'estime de soi des jeunes lors de leur parcours scolaire dans l'enseignement ordinaire a été fortement endommagé par la non considération." Dans le même ordre d'idées, Burnett (1997) a constaté que les affirmations positives faites par les enseignants ont un effet direct sur ce que les élèves disent d'eux-mêmes, ce qui, à son tour, influe directement sur l'estime de soi.

$\mathrm{Au}$ chapitre des pratiques scolaires figurent aussi les difficultés d'apprentissage éprouvées par les élèves, ce qu'Audrit qualifie de problèmes d'ordre scolaire. Autrement dit, les lacunes dans les disciplines scolaires, telles que la langue d'enseignement et les mathématiques, influent négativement sur la construction du concept de soi.

Aux problèmes scolaires s'ajoutent des problèmes de type relationnel, dus principalement au manque d'encouragement et de motivation à l'apprentissage.

Comme facteurs du cadre familial, des auteurs (Audrit, 2015-2016 ; Bouissou, 2001 ; Mensa-Schrèque, 2012) retiennent l'influence de la relation parent-enfant. Lorsque celle-ci est positive, soutenue et régulière, lorsque l'enfant est encouragé et accompagné par les parents dans ses apprentissages, cela renforce à la fois la motivation et l'estime de soi scolaire. Dans la même optique, Wigfield et al. (1997) ont constaté que le sentiment de compétence de l'élève se construit à partir de son rendement antérieur et des rétroactions provenant des agents de socialisation, en particulier des enseignants et des 
parents. En plus de la relation parent-enfant, la qualité des relations fraternelles peut aussi avoir un impact sur l'image de soi du jeune (Audrit, 2015-2016).

Sur le plan de l'intégration sociale, Audrit (2015-2016) note qu'une intégration scolaire propice favorise une estime de soi scolaire positive. Pour cet auteur, l'un ne peut aller sans l'autre. "Les jeunes ont besoin d'une bonne intégration sociale pour une bonne estime de soi scolaire et l'intégration sociale ne peut être possible lorsqu'il y a des difficultés d'apprentissage" (Audrit, 2015-2016, p. 77). Le même constat est fait par Maltais et Herry (1997).

Dans une étude visant à évaluer les déterminants du concept de soi scolaire, l'estime de soi et le stress scolaire, Michie, Glachan et Bray (2001) ont trouvé que l'âge, le genre, les expériences scolaires passées et les motivations à participer à l'enseignement jouent sur l'estime de soi global, le concept de soi scolaire et le stress scolaire. S'agissant particulièrement de l'âge, les élèves jeunes tendent à surestimer leurs compétences du fait qu'ils n'ont pas encore de maturité cognitive nécessaire à l'évaluation critique de leurs habiletés (Manning, 2007).

Pour le genre, Wylie, citée par Marsh, Parker et Barnes (1985), a conclu, à l'issue de l'examen approfondi des recherches menées aux Etats-Unis avant 1977, qu'il n'y avait aucune preuve de la différence de genre dans le concept de soi, et ce, quel que soit l'âge. En revanche, plusieurs études australiennes ont trouvé des différences significatives, lesquelles peuvent dépendre de l'âge, de la composante et de l'instrument de mesure du concept de soi (Marsh \& Smith, 1982). Les études menées particulièrement avec le "Self Description Questionnaire" ont mis en évidence de grandes différences entre les genres en ce qui concerne les aptitudes physiques (en faveur des garçons) et la lecture (en faveur des filles) (Marsh, Parker, \& Barnes, 1985).

Le redoublement fait partie des expériences scolaires passées qui influent sur le concept de soi (Badolo, 2012). Suivant la synthèse de 800 métaanalyses effectuée par Hattie (2009), les non redoublants obtiennent de meilleurs résultats que les redoublants en ce qui concerne l'adaptation sociale et émotionnelle, le concept personnel et l'attitude envers l'école.

Par ailleurs, plusieurs études ont mis en évidence une relation positive entre les attentes de l'enseignant et le concept de soi des élèves (Eder, 1983; Harris, Rosenthal, \& Snodgrass, 1986; Karper \& Martinek, 1982; Milgrim, 1983; Pépin, 1990). Pour Morency (1993), l'élève croit qu'il peut améliorer sa réussite, si l'enseignant est convaincu de sa capacité. De même, suivant l'étude réalisée par Forgette-Giroux et Richard (1995), les attentes des enseignants et des parents constituent la composante du climat psychosocial qui influence le plus le concept de soi et la confiance en soi des élèves. 
Outre les attentes des enseignants, Burnett (2003) a analysé les relations entre le feedback des enseignants et le concept de soi des élèves en mathématiques et en lecture. En recourant à la modélisation par équation structurelle, il a remarqué que l'effet du feedback sur le concept de soi en mathématiques et en lecture est médié par ce que les élèves disent d'euxmêmes.

Des études ont également relevé l'impact de la relation élève-enseignant sur le concept de soi. Hargrave et al. (2016), par exemple, ont observé que les expériences négatives dans cette relation étaient les plus associées au concept de soi scolaire. Quant à MacFarland, Murray et Phillipson (2016), ils ont trouvé que pour les garçons, la proximité avec les enseignants ne permet pas de prédire le concept de soi, alors que le conflit dans la relation élèveenseignant affecte négativement cette variable. En revanche, pour les filles, la proximité et le conflit prédisent le concept de soi, le conflit ayant un plus grand effet.

Le concept de soi est également influencé par les méthodes d'enseignement. A ce sujet, dans une étude effectuée en Suède sur des élèves de $7^{\text {ème }}$ année, âgés de treize ans, Samuelsson (2008) a montré que le travail traditionnel et la résolution des problèmes sont plus efficaces pour le concept de soi des élèves que le travail indépendant. A son tour, Suk (2005) a étudié l'effet de l'approche constructiviste de l'enseignement sur le rendement scolaire, le concept de soi et les stratégies d'apprentissage. Basée sur la démarche expérimentale, l'étude de Suk a révélé que l'approche constructiviste, bien que préférée par les élèves, n'affecte pas le concept de soi mieux que l'enseignement traditionnel. Nawaz, Atta et Khan (2015) ont, quant à eux, étudié au Pakistan, l'effet comparatif de l'apprentissage coopératif et de l'apprentissage démonstratif sur le concept de soi des élèves de $5^{\text {ème }}$ année primaire. Ils ont observé que l'apprentissage coopératif influe mieux sur le concept de soi scolaire que l'approche démonstrative.

\section{Questions de recherche}

Cette étude ambitionne de répondre aux questions suivantes :

a) Quelle est la relation entre le concept de soi en lecture et le concept de soi en mathématiques au niveau de l'élève et de la classe?

b) Dans quelle mesure les caractéristiques de l'élève expliquent-elles les différences dans (a) le concept de soi en lecture et (b) le concept de soi en mathématiques?

c) Dans quelle mesure les caractéristiques de la classe expliquent-elles les différences dans (a) le concept de soi en lecture et (b) le concept de soi en mathématiques après le contrôle des caractéristiques de l'élève? 


\section{Méthodologie \\ Echantillon d'étude}

Les données exploitées dans cette étude proviennent d'une recherche longitudinale réalisée de 2010 à 2014 par le Service de Planification et d'Evaluation en Education de l'Université de Kisangani (République Démocratique du Congo) en partenariat avec le Center for Educational Effectiveness Research de la KU Leuven (Belgique). Axée sur l'efficacité des écoles primaires des deux plus grandes villes (Bunia et Kisangani) de la Province Orientale en République Démocratique du Congo, cette recherche a procédé au suivi, de la $4^{\text {ème }}$ à la $6^{\text {ème }}$ année primaire, d'un échantillon constitué au départ de 4787 élèves issus de 80 classes et 49 écoles (cf. tableau 1).

Pour la réalisation de la recherche, l'échantillonnage des écoles s'est basé sur la structure du système éducatif de la République Démocratique du Congo, laquelle comprend trois réseaux : les écoles publiques non conventionnées (EPUNC), les écoles publiques conventionnées (EPUC) et les écoles privées (EPR). Ces écoles sont respectivement gérées par l'Etat (pour les EPUNC), les Eglises (pour les EPUC) et leurs promoteurs (pour les EPR) qui sont, de manière générale, des particuliers ou des associations. Du fait de cette structure, l'échantillonnage a porté dans un premier temps sur le choix aléatoire, dans chaque réseau, d'un nombre proportionnel d'écoles. Ensuite, dans une école ayant une ou deux classes de $4{ }^{\text {ème }}$ année, ces dernières ont d'office été sélectionnées pour la recherche. En revanche, pour une école ayant plus de deux classes de $4^{\text {ème }}$ année, seulement deux ont été sélectionnées aléatoirement pour faire partie de l'échantillon. Enfin, tous les élèves des classes sélectionnées ont été retenus pour la recherche. Ce sont les données collectées sur les élèves de $4{ }^{\text {ème }}$ année primaire qui ont été analysées dans la présente étude.

Tableau 1. Echantillon d'étude

\begin{tabular}{cccc}
\hline Ville & Nombre d'écoles & $\begin{array}{c}\text { Nombre de } \\
\text { classes }\end{array}$ & Nombre d'élèves \\
\hline Bunia & 13 & 21 & 1516 \\
Kisangani & 36 & 59 & 3271 \\
Total & 49 & 80 & 4787 \\
\hline
\end{tabular}

\section{Instruments de mesure}

Les données analysées dans cette étude ont été collectées au moyen de cinq instruments : (a) un questionnaire de renseignements sur l'élève, (b) un questionnaire d'auto-description de l'élève, (c) un test de lecture compréhension et $(\mathrm{d})$ deux tests de mathématiques.

Comprenant 35 items, le questionnaire de renseignements sur l'élève explore ses caractéristiques démographiques, socio-familiales et son passé scolaire. 
Le questionnaire d'auto-description est extrait de la version française du "Self Description Questionnaire I" (Dierendonck, 2008) développé par Marsh (1988). Des 48 items de type Likert, la version appliquée en $4^{\text {ème }}$ année primaire comprend cinq échelles : (a) le rapport à l'école (10 items), (b) le concept de soi en lecture (10 items), (c) le concept de soi en mathématiques (10 items), (d) les relations avec les parents (9 items) et (e) les relations avec les autres élèves ( 9 items). Au vu de l'étude de la fidélité de ces échelles, la dimension "relations avec les parents" $(\alpha=.56)$ n'a pas été prise en compte dans cette étude. Pour les échelles retenues, certains items ont été rejetés à cause de leur faible contribution à la fidélité (cf. variables retenues).

Le test de lecture compréhension consiste, pour l'élève, à lire un texte tiré du livre de $3^{\text {ème }}$ année primaire, et à répondre à dix questions s'y rapportant. Le test de mathématiques appliqué au début de $4{ }^{\text {ème }}$ année primaire comprend 36 items axés sur le programme de $3^{\text {ème }}$ année primaire. Il couvre les rubriques des fractions, des opérations, de la numération et des mesures. Par contre le test de mathématiques administré à la fin de $4^{\text {ème }}$ année primaire comprend 40 items se rapportant aux mêmes rubriques que le prétest.

\section{Variables retenues}

\section{Variables dépendantes}

Le concept de soi en lecture (CSLECT) et le concept de soi en mathématiques (CSMATH) ont été retenus dans cette étude comme variables dépendantes. Ils proviennent d'un extrait de la version française du "Self Description Questionnaire I" et comprennent respectivement 9 items $(\alpha=.60)$ et 8 items $(\alpha=.66)^{1}$. L'analyse exploratoire (cf. tableau 2) montre que ces deux variables sont positivement corrélées $(\mathrm{r}=.39$, $\mathrm{p}<.01)$.

\section{Variables indépendantes}

Variables de l'élève

Au niveau de l'élève douze caractéristiques ont été prises en compte comme variables indépendantes : deux variables démographiques, une variable socio-familiale, une variable de l'expérience scolaire, quatre aspects cognitifs et quatre aspects non cognitifs de l'apprentissage.

Les variables démographiques se rapportent au genre (codé 0 pour la fille et 1 pour le garçon) et à l'âge (variant de 7 à 16 ans).

Quant à la variable socio-familiale, l'étude s'est intéressée au soutien parental (SOUTPAR). Issu du questionnaire de renseignements sur l'élève, le soutien parental est fourni par trois items examinant si, pour la réalisation de

${ }^{1}$ Les items se rapportant à l'échelle du concept de soi en lecture sont du type "J'aime la lecture ; J'ai des bons points en lecture ; Le devoir en lecture est facile pour moi... " De même pour le concept de soi en mathématiques les items du genre "J'aime les mathématiques; J'apprends rapidement les leçons de mathématiques; J'attends avec impatience les leçons de mathématiques....." 
ses devoirs à domicile, l'élève bénéficie de l'aide (a) de sa mère, (b) de son père et (c) de ses frères et sœurs. L'échelle du soutien parental varie par conséquent de 0 (si l'élève ne bénéficie d'aucun appui) à 3 (si l'élève bénéficie du soutien de toutes les trois catégories de membres de la famille).

Concernant l'expérience scolaire, l'étude a modélisé uniquement le redoublement, codé 0 , pour le non redoublement, et 1 , pour le redoublement d'au moins une classe de la $1^{\text {ère }}$ à la $4^{\text {ème }}$ année.

Les aspects cognitifs de l'apprentissage intégrés dans les analyses se rapportent aux scores obtenus par les élèves aux tests de lecture compréhension et de mathématiques appliqués respectivement au début (PRETESTLECT, $\alpha=.75$; PRETESTMATH, $\alpha=.87$ ) et à la fín (POSTESTLECT, $\alpha=.70$; POSTESTMATH, $\alpha=.86$ ) de $4{ }^{\text {ème }}$ année primaire.

Sur le plan non cognitif, les analyses ont porté sur quatre variables : (a) le rapport à l'école (RAPEC, $\alpha=.60)$, (b) la relation avec les autres élèves (RELAUT, $\alpha=.64$ ), (c) le concept de soi initial en lecture (CSILECT, $\alpha=.60$ ) et (d) le concept de soi initial en mathématiques (CSIMATH, $\alpha=.64$ ).

L'analyse exploratoire des données montre qu'il y a une corrélation positive et significative entre la plupart des caractéristiques individuelles et les variables dépendantes (cf. tableau 2).

Tableau 2. Corrélations, moyennes et écart-types des prédicteurs et des variables de concept de soi au niveau élève

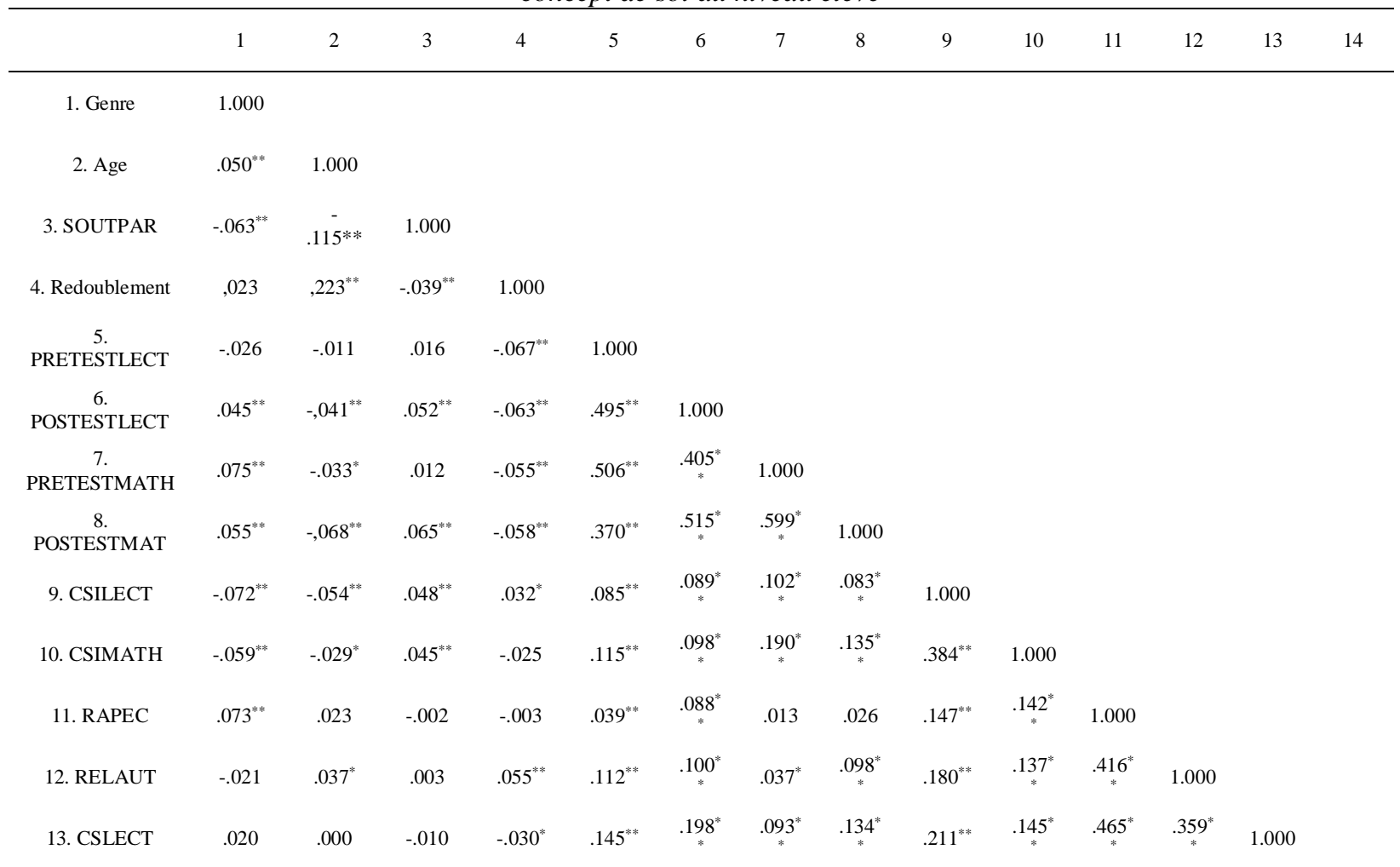




\begin{tabular}{|c|c|c|c|c|c|c|c|c|c|c|c|c|c|c|}
\hline 14. CSMATH & $-.068^{* *}$ & .002 & .003 & .024 & $.056^{* *}$ & $.152^{*}$ & $\begin{array}{c}.094^{*} \\
*\end{array}$ & $.202^{*}$ & $.156^{* *}$ & $.215^{*}$ & $.419^{*}$ & $\begin{array}{c}.372^{*} \\
*\end{array}$ & $\begin{array}{c}.388^{*} \\
*\end{array}$ & 1.000 \\
\hline Moyenne & - & 10.510 & 1.490 & - & 2.650 & 3.250 & $\begin{array}{c}10.99 \\
0\end{array}$ & $\begin{array}{c}11.85 \\
0\end{array}$ & 37.690 & $\begin{array}{c}37.95 \\
0\end{array}$ & $\begin{array}{c}37.95 \\
0\end{array}$ & $\begin{array}{c}33.73 \\
0\end{array}$ & $\begin{array}{c}37.76 \\
0\end{array}$ & $\begin{array}{c}38.44 \\
0\end{array}$ \\
\hline Ecart-type & - & 1.377 & 1.134 & - & 2.502 & 2.670 & 7.325 & 7.966 & 6.154 & 6.168 & 6.091 & 6.340 & 6.176 & 6.304 \\
\hline
\end{tabular}

\section{Variables de la classe}

Trois catégories de variables ont été modélisées au niveau de la classe : (a) les caractéristiques de l'enseignant, (b) la morphologie de la classe et (c) sa composition. Pour les caractéristiques de l'enseignant, outre le genre (codé 0 pour l'enseignante et 1 pour l'enseignant), les analyses ont porté sur son expérience professionnelle. Celle-ci est mesurée par le nombre d'années passées dans l'enseignement et varie de 1 à 43 années.

Concernant la morphologie, l'étude a porté uniquement sur la taille de classe dont l'étendue de variation va de 13 à 89 élèves.

Enfin, six moyennes obtenues par les classes aux tests de lecture compréhension et de mathématiques ainsi que dans les échelles du concept de soi ont été considérées comme des variables de composition. Il s'agit des moyennes obtenues au prétest lecture (MOYPRETESTLECT) et au prétest mathématiques (MOYPRETESTMATH), des moyennes du concept de soi initial en lecture (MOYCSILECT), du concept de soi initial en mathématiques (MOYCSIMATH) et des moyennes des scores aux échelles du rapport à l'école (MOYRAPEC) et de la relation avec les autres élèves (MOYRELAUT).

La plupart des variables indépendantes modélisées au niveau de la classe sont positivement et significativement associées aux indicateurs du concept de soi (cf. tableau 3).

Tableau 3. Corrélations, moyennes et écart-types des prédicteurs et des variables du concept de soi au niveau classe

\begin{tabular}{|c|c|c|c|c|c|c|c|c|c|c|c|}
\hline & 1 & 2 & 3 & 4 & 5 & 6 & 7 & 8 & 9 & 10 & 11 \\
\hline 1. Genre & 1.000 & & & & & & & & & & \\
\hline 2. Expérience & .007 & 1.000 & & & & & & & & & \\
\hline 3. Taille e la classe & -.049 & -.124 & 1.000 & & & & & & & & \\
\hline 4. MOYRAPEC & -.012 & $.231^{*}$ & $-.229^{*}$ & 1.000 & & & & & & & \\
\hline 5. MOYRELAUT & -.026 & .110 & -.218 & $.765^{* *}$ & 1.000 & & & & & & \\
\hline 6. MOYCSILECT & .147 & -.003 & -.108 & $.314^{* *}$ & .179 & 1.000 & & & & & \\
\hline 7. MOYCSIMATH & .110 & -.003 & -.004 & $.368^{* *}$ & $.239^{*}$ & $.823^{* *}$ & 1.000 & & & & \\
\hline $\begin{array}{c}8 . \\
\text { MOYPRETESTLECT }\end{array}$ & -.034 & 206 & $-.248^{*}$ & .147 & .154 & .083 & .056 & 1.000 & & & \\
\hline
\end{tabular}




\begin{tabular}{|c|c|c|c|c|c|c|c|c|c|c|c|}
\hline $\begin{array}{c}9 . \\
\text { MOYPRETESTMATH }\end{array}$ & .074 & .159 & -.014 & .090 & .111 & .145 & .127 & $.640^{* *}$ & 1.000 & & \\
\hline 10. CSLECT & -.095 & $.285^{*}$ & -.211 & $.863^{* *}$ & $.764^{* *}$ & $.278^{*}$ & $.292^{* *}$ & $.298^{* *}$ & $.223^{*}$ & 1.000 & \\
\hline 11. CSMATH & -.074 & .197 & $-.317^{* *}$ & $.811^{* *}$ & $.811^{* *}$ & $.350^{* *}$ & $.357^{* *}$ & .206 & .108 & $.829^{* *}$ & 1.000 \\
\hline Moyenne & - & 18.000 & 53.030 & 34.216 & 31.462 & 34.055 & 31.654 & 2.887 & 11.686 & 37.760 & 38.440 \\
\hline Ecart type & - & 12.755 & 16.724 & 1.96 & 1.937 & 2.199 & 1.915 & 1.575 & 4.432 & 6.176 & 6.304 \\
\hline
\end{tabular}

\section{Analyse des données}

Données manquantes

Puisqu'elle est longitudinale, la recherche d'où proviennent les données exploitées dans cette étude comporte des données manquantes dont le pourcentage varie de $0.2 \%$ à $13 \%$. Pour éviter d'obtenir au niveau de l'échantillon des paramètres qui diffèrent trop de ceux de la population (Graham, 2009), nous avons procédé à l'imputation multiple des données. Cette imputation n'impose aucune hypothèse quant à l'absence de données manquantes (Tabachnick \& Fidell, 2007).

L'imputation des données manquantes a été effectuée au moyen du logiciel R, version 3.5.0, et du package Amelia.

Analyse multiniveau

L'analyse des données a été réalisée suivant un modèle multivarié à deux niveaux, conceptualisé comme un modèle à trois niveaux, dans lequel les scores, dans les échelles du concept de soi en lecture et du concept de soi en mathématiques (niveau 1), sont nichés dans les élèves (niveau 2) qui sont euxmêmes nichés dans les classes (niveau 3). La variation du niveau 1 n'est pas spécifiée dans ce modèle, ce niveau étant utilisé uniquement dans le but de définir la structure multivariée (Goldstein, 2011). En conséquence, l'analyse multiniveau appliquée dans cette étude est basée sur un modèle à deux niveaux : niveau élève et niveau classe. Ce modèle est ainsi formalisé :

$$
Y_{m i j}=\sum_{t=1}^{2} \beta_{0 t} z_{m t}+\sum_{t=1}^{2} \beta_{1 t} z_{m t} X_{m i j}+\sum_{t=1}^{2} \beta_{2 t} z_{m t} W_{m j}+\sum_{t=1}^{2} u_{0 t j} z_{m t}+\sum_{t=1}^{2} \varepsilon_{0 t i j} z_{m t}
$$

Dans lequel $z_{m t}=\left\{\begin{array}{c}1, \text { si } \mathrm{t}=\mathrm{m} \\ 0, \text { autrement }\end{array}\right.$

Dans cette équation, $Y_{m i j}$ est la valeur du résultat $m$ de l'élève $i$ de la classe $j$, avec $m=1,2(1=$ concept de soi en lecture et $2=$ concept de soi en mathématiques), $i=1, \ldots \ldots . ., n_{j}, j=1, \ldots \ldots . j=80$ classes et $t$ des indices de l'ensemble des indicateurs. $\mathrm{X}_{m i j}$ est le vecteur des covariables du niveau élève ; $\mathrm{W}_{m i j}$ est le vecteur des covariables du niveau classe $; \varepsilon_{0 t i j}$ sont les erreurs du niveau élève, lesquelles sont supposées indépendantes à travers les élèves et $u_{0 t j}$ sont des erreurs du niveau classe, également supposées indépendantes à 
travers les classes. L'école n'étant pas intégrée dans le modèle, $u_{0 t j}$ représente également les facteurs non observés à ce niveau.

Les erreurs $\varepsilon_{0 t i j} \mathrm{du}$ niveau élève sont supposées normales multivariées avec des moyennes équivalentes à zéro et la matrice de covariance :

$$
\Sigma=\left[\begin{array}{l}
\varepsilon_{1 i j} \\
\varepsilon_{2 i j}
\end{array}\right], \operatorname{MVN}\left(\left[\begin{array}{l}
0 \\
0
\end{array}\right],\left[\begin{array}{l}
\sigma_{e 1}^{2} \\
\sigma_{e 12} \sigma_{e 2}^{2}
\end{array}\right]\right)
$$

Les erreurs $u_{0 t j} \mathrm{du}$ niveau classe sont également supposées normales multivariées avec des moyennes égales à zéro et la matrice de covariance :

$$
\Omega=\left[\begin{array}{l}
u_{1 j} \\
u_{2 j}
\end{array}\right] \sim \operatorname{MVN}\left(\left[\begin{array}{l}
0 \\
0
\end{array}\right],\left[\begin{array}{l}
\tau_{u 1}^{2} \\
\tau_{u 12} \tau_{u 2}^{2}
\end{array}\right]\right)
$$

L'analyse multiniveau multivariée a été effectuée au moyen du logiciel MLwiN 2.24 (Rasbash et al., 2011). En vue de comparer la taille des effets, nous avons procédé à la standardisation de toutes les variables continues.

Dans le processus d'analyse multiniveau, nous avons, dans un premier temps, spécifié le modèle vide (Modèle 0) afin d'estimer les variances et covariances situées aux niveaux élève et classe. Nous avons ensuite introduit dans le modèle les caractéristiques de l'élève (Modèle 1). Pour ne pas surspécifier ce modèle, le concept de soi initial en lecture et le rendement en lecture ont été modélisés seulement comme prédicteurs du concept de soi en lecture tandis que le concept de soi initial en mathématiques et le rendement en mathématiques n'ont été spécifiés que pour la prédiction du concept de soi en mathématiques. Dans un troisième temps, nous avons introduit les caractéristiques de la classe, en modélisant successivement les caractéristiques de l'enseignant et la morphologie de la classe (Modèle 2) et les variables de composition (Modèle 3). Pour éviter la surspécification du modèle 3, les moyennes de la classe en lecture et dans le concept de soi initial en lecture ont été modélisées pour la prédiction du concept de soi en lecture tandis que les moyennes de la classe en mathématiques et dans le concept de soi initial en mathématiques n'ont été spécifiées que pour la prédiction du concept de soi en mathématiques. Dans la dernière étape (Modèle 4), nous avons introduit toutes les caractéristiques de la classe retenues dans cette étude.

En vue d'apprécier l'ajustement de chaque modèle aux données, par rapport à un modèle moins complet, nous avons utilisé la différence des statistiques de déviance (-2logLikelihood. Cette différence suit la loi du Chi2 à $m$ degrés de liberté, $m$ étant le nombre de paramètres supplémentaires à estimer quand on passe d'un modèle à un autre plus complet (Bressoux, 2010). 


\section{Résultats}

Modèle vide et effet des caractéristiques individuelles

Le tableau 4 reprend, pour le modèle vide, les variances et covariances estimées au niveau élève et au niveau classe. Il indique que la covariance entre le concept de soi en lecture et le concept de soi en mathématiques est positive et significative à chaque niveau du modèle. Le tableau 4 montre en outre que la covariance entre les indicateurs du concept de soi est plus élevée au niveau élève (.417) qu'au niveau classe (.078). Néanmoins, comme le montre le tableau 5, les deux indicateurs du concept de soi scolaire sont plus fortement corrélés au niveau classe $(r=.90)$ qu'au niveau élève $(r=.46)$. Pour chaque indicateur, la variance est quasi exclusivement située au niveau élève (cf. tableau 5 et figure 1$)$.

Tableau 4. Estimation des paramètres pour les effets fixes et aléatoires

\begin{tabular}{|c|c|c|c|c|}
\hline \multirow[b]{2}{*}{ Variable } & \multicolumn{2}{|c|}{$\begin{array}{l}\text { Modèle vide } \\
\text { Modèle } 0\end{array}$} & \multicolumn{2}{|c|}{$\begin{array}{c}\text { Caractéristiques individuelles } \\
\text { Modèle } 1\end{array}$} \\
\hline & CSLECT & CSMATH & CSLECT & CSMATH \\
\hline Intercept & $.021(.036)$ & $.022(.036)$ & $.021(.023)$ & $.029(.024)$ \\
\hline \multicolumn{5}{|l|}{ Niveau Elève } \\
\hline $\begin{array}{l}\text { Genre }(0 \text { : Fille ; } 1 . \\
\text { Garçon })\end{array}$ & & & $.003(.024)$ & $-.028(.024)$ \\
\hline Age & & & $-.010(.012)$ & $-.012(.013)$ \\
\hline SOUTPAR & & & $-.012(.012)$ & $.000(.012)$ \\
\hline Redoublement & & & $-.045(.024)$ & $-.024(.024)$ \\
\hline RAPEC & & & $.420(.014)$ & .381 (.014) \\
\hline RELAUT & & & $.262(.014)$ & $\mathbf{3 0 9}(.014)$ \\
\hline PRETESTLECT & & & $.017(.013)$ & \\
\hline POSTESTLECT & & & $\mathbf{. 0 4 8}(.012)$ & \\
\hline CSILECT & & & $\mathbf{. 0 3 2}(.012)$ & \\
\hline PRETESTMATH & & & & $.013(.013)$ \\
\hline POSTESTMATH & & & & $\mathbf{. 0 2 6}(.013)$ \\
\hline CSIMATH & & & & $\mathbf{. 0 2 6}(.012)$ \\
\hline \multicolumn{5}{|c|}{$\begin{array}{l}\text { Matrice de covariance } \\
\text { intra-classe }\end{array}$} \\
\hline CSLECT & $\mathbf{. 9 1 3}(.019)$ & & $\mathbf{. 6 0 2}(.013)$ & \\
\hline CSMATH & $\mathbf{. 4 1 7}(.015)$ & $\mathbf{. 9 1 9}(.019)$ & .106 (.009) & $\mathbf{. 6 0 4}(.013)$ \\
\hline \multicolumn{5}{|c|}{$\begin{array}{l}\text { Matrice de covariance } \\
\text { interclasse }\end{array}$} \\
\hline CSLECT & $\mathbf{. 0 8 8}(.017)$ & & $\mathbf{. 0 1 1}(.003)$ & \\
\hline CSMATH & $.078(.015)$ & $\mathbf{. 0 8 6}(.016)$ & $.007(.003)$ & $\mathbf{. 0 1 6}(.004)$ \\
\hline -2log-likelihood & 24654.19 & & 21611.99 & \\
\hline
\end{tabular}

Légende : Gras = significatif à au moins 5\%. 
Tableau 5. Modèle nul multiniveau multivarié pour les indicateurs du concept de soi scolaire

\begin{tabular}{ccccccc}
\hline & \multicolumn{3}{c}{ Corrélations } & \multicolumn{2}{c}{ Pourcentage de variance } \\
\cline { 2 - 7 } & \multicolumn{2}{c}{$\begin{array}{c}\text { Niveau } \\
\text { élève }\end{array}$} & $\begin{array}{c}\text { Niveau } \\
\text { classe }\end{array}$ & $\begin{array}{c}\text { Niveau } \\
\text { élève }\end{array}$ & $\begin{array}{c}\text { Niveau } \\
\text { classe }\end{array}$ \\
\hline Indicateurs & 1 & 2 & 1 & 2 & & \\
CSLECT & 1 & & 1 & & $91.2 \%$ & $8.8 \%$ \\
CSMATH & .46 & 1 & .90 & 1 & $91.4 \%$ & $8.6 \%$ \\
\hline
\end{tabular}

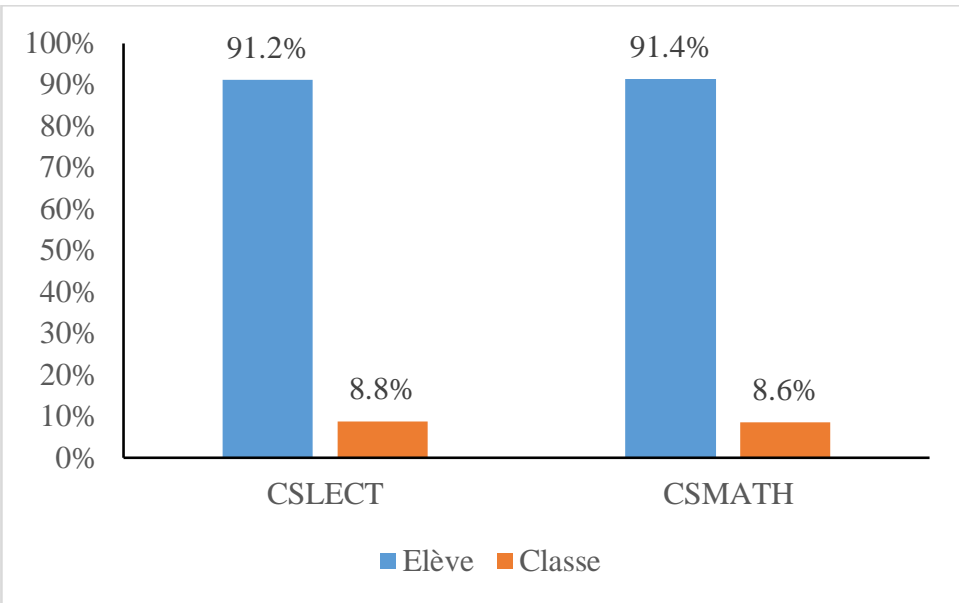

Figure 1. Décomposition de la variance totale dans le modèle vide

La modélisation des caractéristiques individuelles (cf. Modèle 1) montre que le genre, l'âge, le soutien parental et le redoublement n'influent ni sur le concept de soi en lecture ni sur le concept de soi en mathématiques. Il en est autant du niveau cognitif initial en lecture (PRETESTLECT) et en mathématiques (PRETESTMATH). Par contre, le score à l'échelle du rapport à l'école et le score à l'échelle de la relation avec les autres élèves impactent positivement les deux indicateurs du concept de soi scolaire. Le fait qu'un élève se considère fort et aime toutes les matières scolaires affecte plus les indicateurs du concept de soi scolaire que le fait de penser qu'il est en bons termes avec les autres élèves. De même, le rendement en lecture et le rendement en mathématiques obtenus à la fin de l'année scolaire s'avèrent des prédicteurs positifs et significatifs respectivement du concept de soi en lecture $(\mathrm{p}<.001)$ et du concept de soi en mathématiques $(\mathrm{p}<.05)$. Pour chaque variable dépendante, l'indicateur initial correspondant constitue un prédicteur significatif.

Pour le concept de soi en lecture, les caractéristiques individuelles modélisées dans cette étude expliquent $34.1 \%$ de la variance élève, soit $31.1 \%$ de la variance totale située au niveau élève. Elles expliquent $87.5 \%$ de la variance classe, soit $7.7 \%$ de la variance totale située au niveau classe. Ce sont pratiquement les mêmes proportions de la variance intra-classe et interclasse 
qui sont expliquées par les caractéristiques individuelles au niveau du concept de soi en mathématiques (cf. figure 2 et tableau 7).

Le modèle 1 ajuste mieux les données que le modèle vide, la statistique chi-carré étant hautement significative $\left(\chi^{2}=3042.20, \mathrm{dl}=18, \mathrm{p}<.0001\right)$.

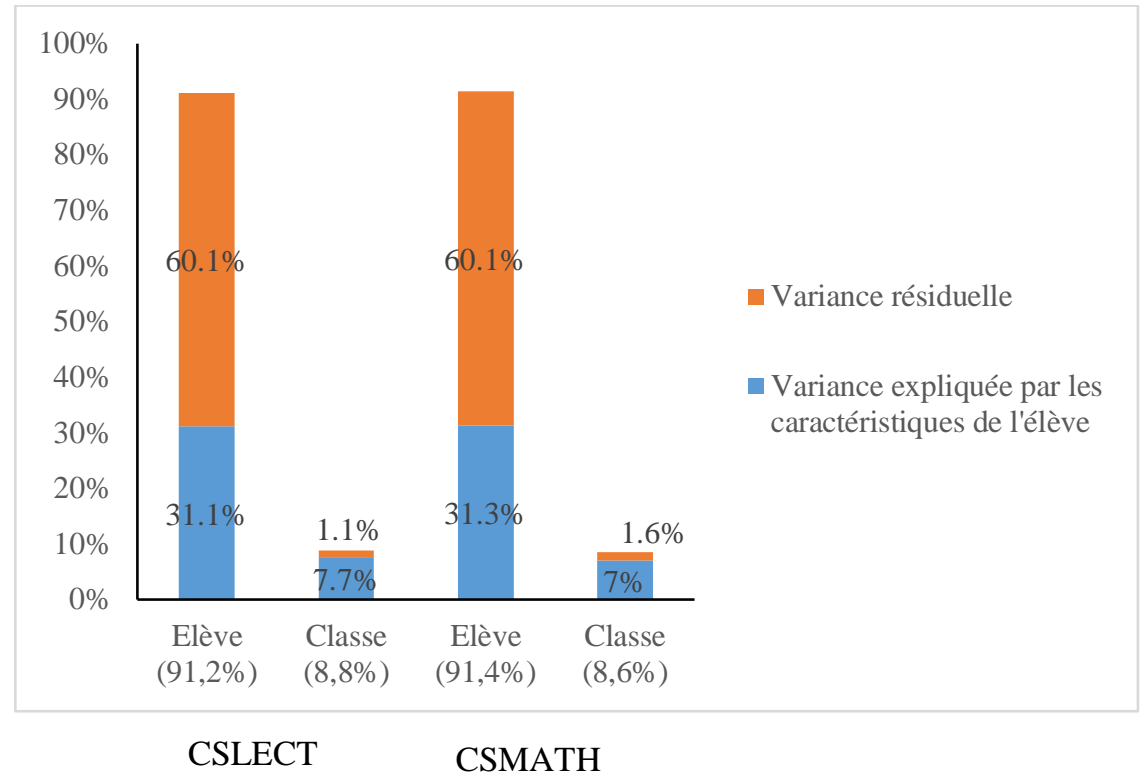

Figure 2. Variance expliquée par les caractéristiques de l'élève

\section{Effet des caractéristiques de la classe}

De la modélisation des caractéristiques de l'enseignant et de la morphologie de la classe (cf. Modèle 2), il ressort que si l'expérience professionnelle est positivement associée au concept de soi en lecture, elle n'influe pas sur le concept de soi en mathématiques. La variation d'un écarttype sur l'échelle de l'expérience professionnelle de l'enseignant s'accompagne de la variation de .04 écart-type sur l'échelle du concept de soi en lecture. En outre, le modèle 2 montre que le genre de l'enseignant et la taille de la classe n'affectent aucun indicateur du concept de soi. En dépit de ce résultat, la modélisation des caractéristiques individuelles, de celles de l'enseignant et de la morphologie de la classe (Modèle 2) s'ajuste aux données mieux que la seule modélisation des caractéristiques de l'élève $\left(\chi^{2}=12.82\right.$, dl $=6, \mathrm{p}<.05)$. 
Tableau 6. Estimation des paramètres pour les effets fixes et aléatoires

\begin{tabular}{|c|c|c|c|c|c|c|}
\hline \multirow[b]{2}{*}{ Variable } & \multicolumn{2}{|c|}{$\begin{array}{c}\text { Caractéristiques individuelles+ } \\
\text { Caractéristiques de l'enseignant et } \\
\text { morphologie de la classe } \\
\text { Modèle } 2 \\
\end{array}$} & \multicolumn{2}{|c|}{$\begin{array}{c}\text { Caractéristiques individuelles+ } \\
\text { Composition de la classe } \\
\text { Modèle } 3\end{array}$} & \multicolumn{2}{|c|}{$\begin{array}{c}\text { Caractéristiques individuelles+ } \\
\text { toutes les caractéristiques de la } \\
\text { classe } \\
\text { Modèle } 4\end{array}$} \\
\hline & CSLECT & CSMATH & CSLECT & CSMATH & CSLECT & CSMATH \\
\hline Intercept & $.057(.030)$ & $.051(.034)$ & $.024(.022)$ & $.029(.024)$ & $.058(.028)$ & $.048(.034)$ \\
\hline Niveau Elève & & & & & & \\
\hline $\begin{array}{l}\text { Genre }(0: \text { Fille ; } 1 \text { : } \\
\text { Garçon) }\end{array}$ & $.006(.024)$ & $-.024(.024)$ & $.005(.024)$ & $-.028(.024)$ & $.007(.024)$ & $-.023(.024)$ \\
\hline Age & $-.010(.012)$ & $-.014(.013)$ & $-.011(.012)$ & $-.015(.013)$ & $-.011(.012)$ & $-.016(.013)$ \\
\hline SOUTPAR & $-.011(.012)$ & $.000(.012)$ & $-.012(.012)$ & $.000(.012)$ & $-.011(.012)$ & $.000(.012)$ \\
\hline Redoublement & $-.043(.024)$ & $-.023(.024)$ & $-.044(.024)$ & $-.025(.024)$ & $-.043(.024)$ & $-.023(.024)$ \\
\hline RAPEC & $\mathbf{. 4 2 0}(.014)$ & $\mathbf{3 8 0}(.014)$ & $.411(.014)$ & $.379(.014)$ & $.411(.014)$ & $\mathbf{. 3 7 8}(.014)$ \\
\hline RELAUT & $.262(.014)$ & $.308(.014)$ & $.261(.014)$ & $.303(.014)$ & $.261(.014)$ & $.303(.014)$ \\
\hline PRETESTLECT & $.016(.013)$ & & $.004(.014)$ & & $.004(.014)$ & \\
\hline POSTESTLECT & $\mathbf{. 0 4 7}(.012)$ & & $\mathbf{. 0 4 5}(.012)$ & & $\mathbf{. 0 4 5}(.012)$ & \\
\hline CSILECT & $\mathbf{. 0 3 3}(.012)$ & & $\mathbf{. 0 3 0}(.012)$ & & $\mathbf{. 0 3 0}(.012)$ & \\
\hline PRETESTMATH & & $.013(.013)$ & & $.022(.014)$ & & $.022(.014)$ \\
\hline POSTESTMATH & & $.025(.013)$ & & $.026(.013)$ & & $\mathbf{. 0 2 6}(.013)$ \\
\hline CSIMATH & & $\mathbf{. 0 2 6}(.012)$ & & $\mathbf{. 0 2 3}(.012)$ & & $.023(.012)$ \\
\hline Niveau classe & & & & & & \\
\hline $\begin{array}{l}\text { Genre enseignant }(0 \text { : } \\
\text { Enseignante } ; 1: \\
\text { Enseignant })\end{array}$ & $-.057(.033)$ & $-.030(.038)$ & & & $-.056(.030)$ & $-.027(.038)$ \\
\hline Expérience du Maître & $.042(.016)$ & $.014(.019)$ & & & $\mathbf{. 0 3 0}(.015)$ & $.017(.019)$ \\
\hline Taille de la classe & $-.005(.017)$ & $-.036(.020)$ & & & $.005(.016)$ & $-.028(.020)$ \\
\hline MOYRAPEC & & & $.076(.025)$ & $.003(.029)$ & $.066(.024)$ & $-.007(.030)$ \\
\hline MOYRELAUT & & & $-.023(.023)$ & $.045(.028)$ & $-.019(.022)$ & $.044(.028)$ \\
\hline MOYCSILECT & & & $-.003(.017)$ & & $.004(.016)$ & \\
\hline MOYPRETESTLECT & & & $.027(.018)$ & & $.024(.018)$ & \\
\hline MOYCSIMATH & & & & $.020(.019)$ & & $.024(.019)$ \\
\hline MOYPRETESTMATH & & & & $-.030(.020)$ & & $-.031(.020)$ \\
\hline $\begin{array}{l}\text { Matrice de covariance } \\
\text { intra-classe }\end{array}$ & & & & & & \\
\hline CSLECT & $\mathbf{6 0 2}(.013)$ & & $.602(.013)$ & & $\mathbf{. 6 0 2}(.013)$ & \\
\hline CSMATH & $\mathbf{. 1 0 6}(.009)$ & $\mathbf{. 6 0 4}(.013)$ & $\mathbf{. 1 0 6}(.009)$ & $.604(.013)$ & $\mathbf{. 1 0 6}(.009)$ & $\mathbf{. 6 0 4}(.013)$ \\
\hline $\begin{array}{l}\text { Matrice de covariance } \\
\text { interclasse }\end{array}$ & & & & & & \\
\hline CSLECT & $.008(.003)$ & & $.007(.003)$ & & $.005(.002)$ & \\
\hline CSMATH & $.005(.003)$ & $.015(.004)$ & $.006(.002)$ & $.014(.004)$ & $.005(.002)$ & $\mathbf{. 0 1 3}(.004)$ \\
\hline -2log-likelihood & 21599.17 & & 21587.35 & & 21577.95 & \\
\hline
\end{tabular}

Légende : Gras = significatif à au moins $5 \%$.

En introduisant dans l'analyse les variables de composition (cf. Modèle 3), il n'y a que la moyenne des scores à l'échelle du rapport à l'école qui impacte positivement le concept de soi en lecture. Au regard de cette modélisation, aucune variable de composition de classe n'influe significativement sur le concept de soi en mathématiques. Pourtant le modèle 3 ajuste mieux les données que le modèle $1\left(\chi^{2}=24.64, \mathrm{dl}=8, \mathrm{p}<.01\right)$.

En modélisant toutes les caractéristiques de la classe retenues dans cette étude (cf. Modèle 4), il s'avère que l'expérience professionnelle des enseignants impacte positivement le concept de soi en lecture et non le concept de soi en mathématiques. Des variables de composition de classe modélisées dans cette étude, seule la moyenne des scores à l'échelle du rapport à l'école 
affecte positivement le concept de soi en lecture. Ainsi, contrairement au concept de soi en lecture, le concept de soi en mathématiques n'est affecté par aucune variable de la classe. Le modèle 4 ajuste les données mieux que le modèle $1\left(\chi^{2}=34.04, \mathrm{dl}=14, \mathrm{p}<.05\right)$ et le modèle vide $\left(\chi^{2}=3076.24, \mathrm{dl}=\right.$ $32, \mathrm{p}<.001)$.

L'ensemble des caractéristiques individuelles et de la classe explique au moins $34.1 \%$ et $83.7 \%$ respectivement de la variance intra-classe et de la variance interclasse (cf. tableau 7). Au niveau de la classe, ces caractéristiques expliquent $0.6 \%$ et $0.3 \%$ de la variance totale respectivement pour le concept de soi en lecture et le concept de soi en mathématiques (cf. figure 3 ).

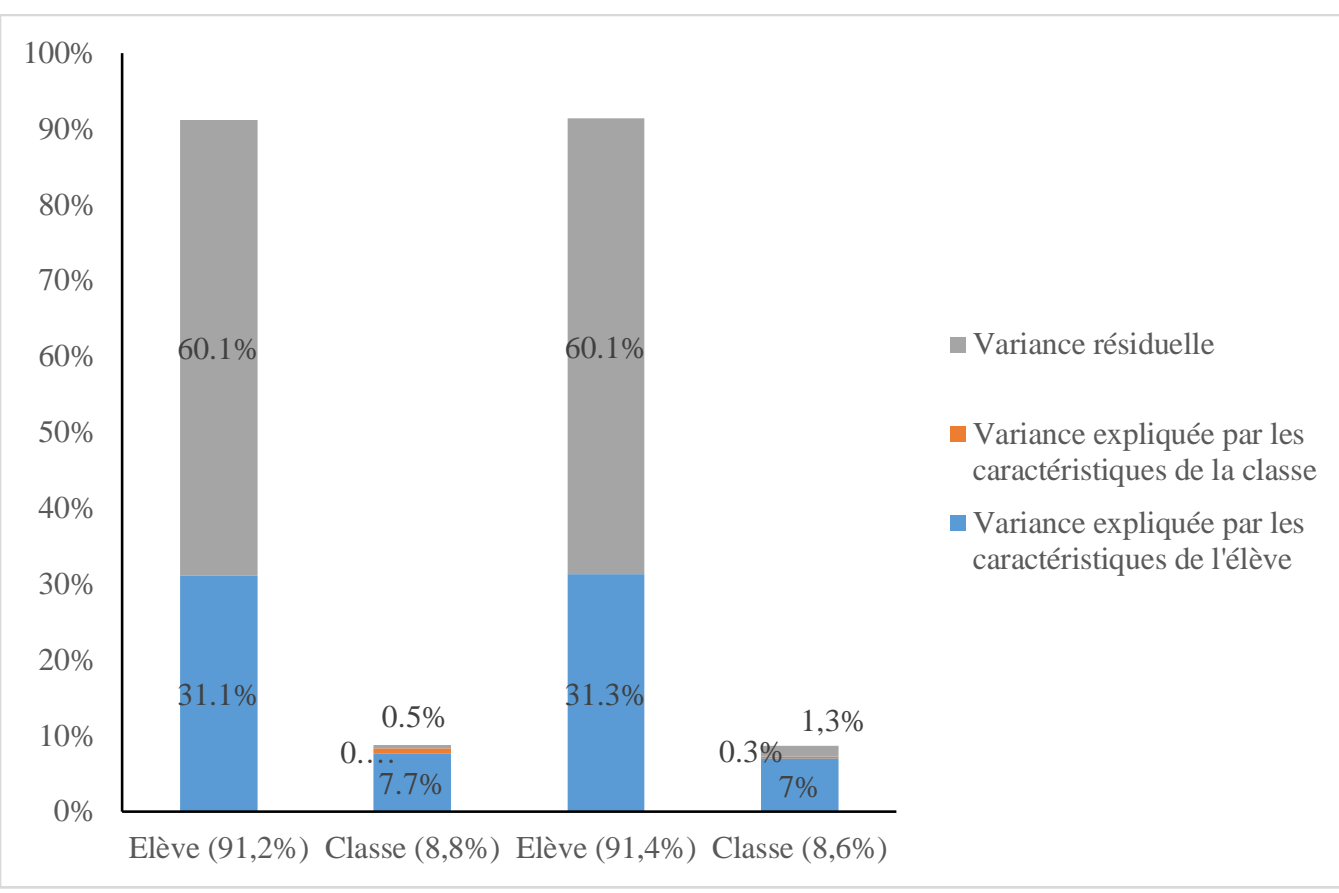

CSLECT

CSMATH

Figure 3. Variance expliquée par les caractéristiques de l'élève et de la classe 
Tableau 7. Variance des deux indicateurs de soi scolaire expliquée à deux niveaux

\begin{tabular}{|c|c|c|c|c|c|}
\hline & $\begin{array}{l}\text { Décompositio } \\
\mathrm{n} \\
\text { de la variance } \\
\text { totale }\end{array}$ & $\begin{array}{l}\text { Variance } \\
\text { expliquée par } \\
\text { les } \\
\text { caractéristiqu } \\
\text { es de l'élève }\end{array}$ & $\begin{array}{l}\text { Variance } \\
\text { expliquée par } \\
\text { les } \\
\text { caractéristiqu } \\
\text { es de l'élève, } \\
\text { de } \\
\text { l'enseignant } \\
\text { et la } \\
\text { morphologie } \\
\text { de la classe }\end{array}$ & $\begin{array}{l}\text { Variance } \\
\text { expliquée par } \\
\text { les } \\
\text { caractéristiqu } \\
\text { es de l'élève } \\
\text { et les } \\
\text { variables de } \\
\text { composition } \\
\text { de classe }\end{array}$ & $\begin{array}{l}\text { Variance } \\
\text { expliquée par } \\
\text { les } \\
\text { caractéristiqu } \\
\text { es de l'élève } \\
\text { et de toutes } \\
\text { les } \\
\text { caractéristiqu } \\
\text { es de la classe }\end{array}$ \\
\hline Niveau & Modèle 0 & Modèle 1 & Modèle 2 & Modèle 3 & Modèle 4 \\
\hline \multicolumn{6}{|l|}{ CSLECT } \\
\hline Elève & $91.2 \%$ & $34.1 \%$ & $34.1 \%$ & $34.1 \%$ & $34.1 \%$ \\
\hline Classe & $8.8 \%$ & $87.5 \%$ & $90.9 \%$. & $92.0 \%$ & $94.3 \%$ \\
\hline \multicolumn{6}{|l|}{ CSMATH } \\
\hline Elève & $91.4 \%$ & $34.3 \%$ & $34.3 \%$ & $34.3 \%$ & $34.3 \%$ \\
\hline Classe & $8.6 \%$ & $81.4 \%$ & $82.6 \%$ & $83.7 \%$ & $83.7 \%$ \\
\hline
\end{tabular}

\section{Discussion des résultats}

Sur la base du modèle multiniveau multivarié, cette étude a analysé l'impact des caractéristiques de l'élève et de la classe sur le concept de soi. Elle a mis en évidence le fait que le concept de soi en lecture et le concept de soi en mathématiques sont fortement corrélés mais sont différemment associés aux caractéristiques de l'élève et de la classe. A l'instar de la plupart des recherches antérieures, cette étude a montré que le rendement des élèves dans le domaine cognitif affecte le concept de soi scolaire (Audrit, 2015-2016). En outre, l'étude a montré que l'âge et le genre n'influent ni sur le concept de soi en lecture, ni sur le concept de soi en mathématiques, contredisant ainsi le constat relevé par les études d'Audrit (2015-2016) et de Michie, Glachan et Bray (2001).

Concernant l'effet du genre, en conformité avec les recherches américaines examinées par Wylie, citée par Marsh, Parker et Barnes (1985), le résultat mis en évidence par cette étude, diffère de l'observation relevée par les recherches australiennes selon lesquelles il y a d'importantes différences entre les garçons et les filles dans le concept de soi en lecture (en faveur des filles) et des faibles différences dans les autres domaines du concept de soi (Marsh, Parker, \& Barnes, 1985).

Le redoublement n'a pas non plus impacté les deux indicateurs du concept de soi examinés dans cette étude. Ce résultat contredit la conclusion des travaux intégrés dans les méta-analyses synthétisées par Hattie (2009). De même, contrairement aux observations réalisées par Audrit (2015-2016), Bouissou (2001) et Mensa-Schrèque (2012), le soutien parental n'influe pas sur le concept de soi. Ce résultat remet en question la qualité du soutien parental dont ont bénéficié les élèves impliqués dans cette recherche. 
Cette étude a également souligné l'impact des relations qu'un élève entretient avec les autres élèves sur le concept de soi. Ce résultat est conforme avec le résultat de l'étude menée par Audrit (2015-2016) selon lequel les facteurs de type relationnel influent sur la construction du concept de soi. En plus, les analyses effectuées ont révélé l'effet positif et significatif du rapport à l'école sur les deux indicateurs du concept de soi, pris en compte dans cette étude.

Au niveau de la classe, les analyses effectuées dans cette étude ont montré que l'expérience professionnelle des enseignants affecte positivement le concept de soi en lecture et non le concept de soi en mathématiques. Ce résultat soulève une question intéressante pour les recherches ultérieures. Il importera en effet de comprendre pourquoi, et dans quelles circonstances, l'expérience professionnelle affecte la construction du concept de soi en lecture et non celle du concept de soi en mathématiques.

Comme effet de composition, seule la moyenne des scores à l'échelle du rapport à l'école constitue un bon prédicteur du concept de soi en lecture. Ici également, il convient de comprendre pourquoi cette variable de composition est associée uniquement au concept de soi en lecture.

En dépit de la méthodologie adoptée, cette étude comporte quelques limites. La première tient au fait que le modèle 1 explique seulement $31.1 \%$ et $31.3 \%$ de la variance totale située au niveau élève respectivement pour le concept de soi en lecture et le concept de soi en mathématiques. Ces résultats indiquent que des caractéristiques individuelles les plus pertinentes pour prédire ces indicateurs du concept de soi n'ont pas été modélisées dans cette étude. Il s'agit des variables telles que la relation parent-élève (Audrit, 20152016) ; Bouissou, 2001 et Mensa-Schrèque, 2012), les difficultés d'apprentissage (Audrit, 2015-2016), les expériences scolaires passées et la motivation à l'apprentissage (Michie, Glachan, \& Bray, 2001).

En outre, bien que le modèle 4 explique $94.3 \%$ de la variance entre classe pour le concept de soi en lecture et $83.7 \%$ de la variance entre classe pour le concept de soi en mathématiques, cette étude n'a pas modélisé les caractéristiques de la classe qui impactent le mieux le concept de soi scolaire. Ces caractéristiques se rapportent particulièrement aux relations enseignantélèves (Hargrave et al., 2016 ; MacFarland, Murray, \& Phillipson, 2016), aux attentes des enseignants sur les capacités des élèves (Burnett, 1997 ; Eder, 1983; Harris, Rosenthal, \& Snodgrass, 1986; Karper \& Martinek, 1982; Milgrim, 1983; Pépin, 1990), au feedback que ces derniers réalisent au cours $\mathrm{du}$ processus enseignement-apprentissage (Burnett, 2003) et aux méthodes d'enseignement (Nawaz, Atta, \& Khan,2015 ; Samuelsson, 2008 ; Suk, 2005).

Comme deuxième limite, cette étude n'a pas analysé les relations indirectes entre les variables indépendantes et dépendantes. Il est par exemple logique de penser que l'expérience professionnelle de l'enseignant affecte le 
concept de soi des élèves par la médiation des variables telles que le rendement scolaire, le feedback, les attentes de l'enseignant et les méthodes d'enseignement.

Comme troisième limite, cette étude n'a pas permis d'établir si la moyenne des scores à l'échelle du rapport à l'école affecte le concept de soi en lecture de façon homogène pour tous les élèves ou de manière plutôt différentielle.

Ces limites constituent des points intéressants sur lesquels la recherche ultérieure pourrait bien s'appuyer pour mieux analyser la relation entre, d'une part, les caractéristiques individuelles et de la classe et, d'autre part, le concept de soi.

\section{Conclusion}

Le but de cette étude était d'identifier les caractéristiques individuelles et les caractéristiques de la classe qui impactent le concept de soi des élèves. Pour cela, l'étude a exploité des données collectées auprès d'un échantillon de 4787 élèves de quatrième année primaire répartis dans 80 classes et 49 écoles des villes de Bunia et de Kisangani en République Démocratique du Congo. L'analyse de ces données a été effectuée au moyen de l'analyse multiniveau multivariée.

De la modélisation des caractéristiques de l'élève, il ressort que le genre, l'âge, le soutien parental et le redoublement n'influent ni sur le concept de soi en lecture, ni sur le concept de soi en mathématiques. En revanche, sur le plan cognitif, les scores au test de lecture et au test de mathématiques sont positivement associés respectivement au concept de soi en lecture et au concept de soi en mathématiques. Sur le plan non cognitif, les scores à l'échelle du rapport à l'école et à celle de la relation avec les autres élèves permettent de prédire chaque indicateur du concept de soi retenu dans cette étude.

Au niveau de la classe, aucune variable modélisée dans cette étude n'affecte le concept de soi en mathématiques. Par contre, les enseignants plus expérimentés affectent le concept de soi en lecture mieux que les enseignants moins expérimentés. Enfin, des variables de composition de classe modélisées dans cette étude, seul le score moyen à l'échelle du rapport à l'école influe positivement et significativement sur le concept de soi en lecture.

Etant donné les limites de cette étude, résumées dans la section antérieure, il importe de modéliser, dans l'avenir, des caractéristiques individuelles et des caractéristiques de la classe qui impactent le mieux le concept de soi scolaire, caractéristiques évoquées dans la discussion des résultats de cette étude. Il sera également intéressant d'étudier les relations directes et indirectes, voire des relations réciproques entre les prédicteurs et les critères, en recourant, notamment, aux équations structurelles. Il importe 
aussi d'analyser systématiquement l'effet de composition de classe, relatif au rapport à l'école, afin de déterminer si cet effet est générique ou plutôt différentiel.

\section{Remerciements}

Les auteurs remercient vivement la Coopération universitaire flamande (VLIR-UOS) pour l'appui qu'elle apporte au projet "Contribution à l'amélioration de l'efficacité des écoles primaires et secondaires de la Province Orientale en République Démocratique du Congo".

\section{References:}

1. Alsaker, F. D. (1989). School achievement, perceived academic competence and global self-esteem. School Psychology International, $10,147-158$.

2. Altmann, H. A., \& Dupont, S. F. (1988). The relationship between academic self-concept, global self-concept, and academic achievement. Canadian Journal of Counseling, 22, 170-174.

3. Audrit, G. (2015-2016). La construction de l'estime de soi scolaire chez les adolescents de l'enseignement spécialisé de type 3. (Mémoire de Master en Sciences de l'Education). Université catholique de Louvain.

4. Awad, G. H. (2007). The role of racial identity, academic self-concept, and self-esteem in the prediction of academic outcomes for African American students. Journal of Black Psychology, 33(2), 188-207.

5. Badolo, B. L. (2012). Redoublement et estime de soi chez des élèves burkinabè du primaire. European Scientific Journal, 8(8), 61-72.

6. Bouissou, C. (2001). Dynamique éducative familiale et construction identitaire de l'enfant. Revue internationale de l'éducation familiale, 5(2), 43-61.

7. Bressoux, P. (2010). Modélisation statistique appliquée aux sciences sociales. Bruxelles : De Boeck.

8. Burnett, $P$. C. (1997). Self-esteem and self-talk enhancement in upper primary school children. Research Information for Teachers, 2, 1-4.

9. Burnett, P. C. (2003). The impact of teacher feedback on student selftalk and self-concept in reading and mathematics. The Journal of Classroom Interaction, 38(1), 11-16.

10. Chiu, M. M., \& Klassen, R. B. (2010). Relations of mathematics selfconcept and its calibration with mathematics achievement: Cultural differences among fifteen year-olds in 34 countries. Learning and Instruction, 20, 2-17. 
11. Cokely, K. (2000). An investigation of academic self-concept and its relationships to academic achievement in African American college students. Journal of Black Psychology, 26, 148-164.

12. Creemers, B., \& Kyriakides, L. (2010). Using the dynamic model to develop an evidence-based and theory-driven approach to school improvement. Irish Educational Studies, 29(1), 5-22.

13. Dierendonck, C. (2008). Validation psychométrique d'un questionnaire francophone de description de soi adapté aux préadolescents. Mesure et évaluation en éducation, 31(1), 51-91.

14. Eder, D. (1983). Ability grouping and student's academic selfconcepts: A case study. Elementary School Journal, 84, 149-161.

15. Eguavoen, E. O., \& Eniola, M. S. (2016). Influence of self-concept and social acceptance on academic achievement of students with impairment in Oyo state, Nigeria. International Journal of Arts and Humanities, 5(3), 213-230.

16. Fin, L. S., \& Ishak, Z. (2014). Non-academic self-concept and academic achievement: The indirect effect mediated by academic selfconcept. Research Journal in Organizational Psychology \& Educational Studies, 3(3), 184-188.

17. Forgette-Giroux, R., \& Richard, M. (1995). L'influence du climat psychosocial de l'école et le concept de soi des élèves. Revue canadienne de l'éducation, 20(3), 367-377.

18. Ghazvini, S. D. (2011). Relationships between self-concept and academic performance in high school students. Procedia Social and Behavioral Sciences, 11, 1034-1039.

19. Goldstein, H. (2011). Multilevel statistical models, 4th ed. London, UK: John Wiley \& Sons, Ltd.

20. Graham, J. W. (2009). Missing data analysis: Making it work in the real world. Annual Rewiew of Psychology, 60, 549-576.

21. Hanan, E. A. A. R., Shabana, K., \& Mona, T. E-N. (2017). Relationship between academic self-concept and student'performance among school age children. American Journal of Nursing Science, 5(6), 295-302.

22. Hargrave, L. D., Tyler K. M., Thompson, F., \& Danner, F. (2016). An examination of the association between student-teacher interactions and academic self-concept among African male high school students. Journal of African Males in Education, 7(2), 33-49.

23. Harris, M., Rosenthal, R., \& Snodgrass, S. (1986). The effects of teacher expectations, gender, and behavior on pupil academic performance and self-concept. Journal of Educational Research, 79, 173-179. 
24. Hattie, J. A. C. (2009). Visible learning: A synthesis of over 800 metaanalyses relating to achievement. New York: Routledge.

25. Jirdehi, M. Y., Asgari, F., Tabari, R., \& Leyli, E. K. (2018). Study the relationship between medical sciences students' self-esteem and academic achievement of Guilan University of medical sciences. Journal of Education and Health Promotion, 7, 1-7.

26. Karper, W. B., \& Martinek, T. J. (1982). Differential influence of various instructional factors on self-concepts of handicapped and nonhandicapped children in mainstreamed physical education classes. Perceptual and Motor Skills, 54, 831-835.

27. Keltikangas-Järvinen, L. (1992). Self-esteem as a predictor of future school achievement. European Journal of Psychology of Education, 7, 123-130.

28. Kurtz-Costes, B. E. (1994). Self-concept, attribution beliefs, and school achievement: A longitudinal analysis. Contemporary Educational Psychology, 19, 199-216.

29. Lyon, M. A., \& MacDonald, N. T. (1990). Academic self-concept as a predictor of achievement for a sample of elementary school students. Psychological Report's, 66, 1135-1142.

30. MacFarland, L., Murray, E., \& Phillipson, S. (2016). Student-teacher relationships and student self-concept: Relations with teacher and student gender. Australian Journal of Education, 60(1), 5-25.

31. Maltais, C., \& Herry, Y. (1997). Le concept de soi des élèves éprouvant des difficultés d'apprentissage. Education et Francophonie, 25(2), 1-11.

32. Manning, M. A. (2007). Self-concept and self-esteem in adolescents. Student Services, 11-15.

33. Marsh, H. W. (1984). Relationship among dimensions of selfattribution, dimensions of self-concept and academic achievement. Journal of Educational Psychology, 76, 1291-1380.

34. Marsh, H. W. (1988). Self-description questionnaire: A theoretical and empirical basis for the measurement of multiple dimensions of preadolescent self-concept: A test manual and a research monograph. San Antonio, TX: Psychological Corporation.

35. Marsh, H. W. (1990). A multidimensional, hierarchical model of selfconcept: Theoretical and empirical justification. Educational Psychology Review, 2(2), 77-172.

36. Marsh, H. W., Hau, K. T., \& Kong, C. K. (2002). Multilevel causal ordering of academic self-concept and achievement: Influence of language of instruction (English compared with Chinese) for Hong 
Kong students. American Educational Research Journal, 39(3), 727763.

37. Marsh, H. W., Parker, J., \& Barnes, J. (1985). Multidimensional adolescent self-concepts: Their relationship to age, sex, and academic measures. American Educational Research Journal, 22(3), 422-444.

38. Marsh, H., \& Yeung, A. S. (1997). Causal effects of academic selfconcept on academic achievement: Structural equation of longitudinal data. Journal of Educational Psychology, 89(1), 41-54.

39. Marsh, H. W., \& Smith, I. D. (1982). Multitrait-multimethod analyses of two self-concept instruments. Journal of Educational Psychology, 74, 430-440.

40. Mensa-Schrèque, M-F. (2012). De l'estime de soi à la réussite scolaire : les valeurs éthiques au conseil de coopérative de classe. Paris : L'Harmattan.

41. Michie, F., Glachan, M., \& Bray, D. (2001). An evaluation of factors influencing the academic self-concept, self-esteem and academic stress for direct and re-entry students in higher education. Educational Psychology, 21(4), 455-472.

42. Milgrim, E. L. (1983). Student's perceptions of teacher expectation and attitudes toward reading in three classrooms (Master's thesis). University of Pennsylvania.

43. Morency, L. (1993). Pygmalion en classe : les enseignants accordentils une chance égale d'apprendre à tous leurs élèves? Québec : Presses Interuniversitaires.

44. Nawaz, Q., Atta, M. A., \& Khan, F. (2015). Effect of cooperative learning on the self-concept of high \& low achiever students at elementary level. Gomal University Journal of Research, 31(1), 41-52.

45. O'Mara, A. J., \& Marsh, H. W. (2006). Support for a reciprocal effects model of self-concept and academic achievement through a contrast of multidimensionnal and unidimensionnal approaches. Fourth International Bienal Self Research Conference. Ann Arbor, US, $23^{\text {rd }}$ 26the July, 2006. Proceedings: Self-concept, Motivation Social and Personal Identity for $21^{\text {st }}$ century.

46. Palardy, G., \& Rumberger, R. W. (2008). Teacher effectiveness in first grade: The importance of background qualifications, attitudes and pratices for student learning. Educational Evaluation and Policy Analysis, 30(2), 111-140.

47. Pekrun, R. (1992). The impact of emotions on learning and achievement: Towards a theory of cognitive/motivational mediators. Applied Psychology, 41(4), 359-376. 
48. Pekrun, R., Goetz, T., Titz, W., \& Perry, R. P. (2002). Positive emotions in education. In E. Frydenberg (dir.). Meeting goals, visions, and challenges (149-173). Oxford: Oxford University Press.

49. Pépin, M. (1990). Pygmalion à l'école : synthèse de la littérature (1968-1986) (Thèse de maîtrise inédite). Université d'Ottawa.

50. Rasbash, J., Charlton, C., Browne, W. J., Hearly, M., \& Cameron, B. (2011). MLwiN version 2.24. University of Bristol: Center for Multilevel Modelling.

51. Raynor, J. O., \& McFarlin, D. B. (1986). Motivation and the selfsystem. In R. M. Sorrentino \& E. T. Higgins (dirs.), Handbook of motivation and cognition: Foundations of social behavior (pp. 315349). New York, NY, US: Guilford Press.

52. Rotatori, A. F. (1994). Multidimensional self-concept scale. Measurement and Evaluation in Counseling and Development, 26(4), 265-268.

53. Samuelsson, J. (2008). The impact of different teaching methods on students' arithmetic and self-regulated learning skills. Educational Psychology in Practice, 24(3), 237-250.

54. Scheerens, J. (1990). School effectiveness research and the development of process indicators of school functioning. School Effectiveness and School Improvement, 1(1), 61-80.

55. Scheerens, J., \& Bosker, R. J. (1997). The foundations of educational effectiveness. Oxford, UK: Pergamon.

56. Sénéchal, E. (1997). La relation entre le concept de soi et le rendement scolaire chez les élèves de troisième année primaire (Mémoire de maîtrise inédite). Université Laval.

57. Shavelson, R. J., Hubner, J. J., \& Stanton, G. C. (1976). Validation of construct interpretations. Review of Educational Research, 46, 407441.

58. Skaalvik, E. M., \& Hagtvet, K. A. (1990). Academic achievement and self-concept: An analysis of causal predominance in a developmental perspective. Journal of Personality and Social Psychology, 58, 292307.

59. Spinath, B., Spinath, F., Harlaar, N., \& Plomin, R. (2006). Predicting school achievement from general cognitive ability, self-perceived ability, and intrinsic value. Intelligence, 34, 363-374.

60. Suk, K. J. (2005). The effects of a constructivist teaching approach on student academic achievement, self-concept, and learning strategies. Asia Pacific Education Review, 6, 7-19.

61. Tabachnick, B. G., \& Fidell, L. S. (2007). Using multivariate statistics, 5th edition. Boston: Pearson Education, Inc. 
62. Tripathy, M., \& Srivastava, S. K. (2012). To study the effect of academic achievement on the level of self-confidence. International Journal of Yoga and Allied sciences, 1(1), 33-45.

63. UNESCO (2015). Education pour tous 2000-2015 : progrès et enjeux. Rapport mondial de suivi sur l'EPT 2015. Paris : UNESCO.

64. Uysal, S. (2015). Factors affecting the mathematics achievement of Turkish students in PISA 2012. Educational Research and Reviews, 10(12), 1670-1678.

65. Wigfield, A., Harold, R. D., Freedman-Doan, C., Eccles, J. S., Suk, Y., K., Arbreton, A., \& Blumenfeld, P. C. (1997). Change in children's competence beliefs and subjective task values across the elementary school years. Journal of Educational Psychology, 89, 451-469.

66. Yeung, A. S., Chui, H. S, \& Lau, I. C. (1999). Hierarchical and multidimensional academic self-concept of commercial students. Contemporary Educational Psychology, 24(4), 376-359.

67. Yeung, A. S., McInerney, D. M., \& Russell-Bowie, D. (2001). Hierarchical, multidimensional creative arts self-concept. Australian Journal of Psychology, 53(3), 125-133. 